\title{
LA-6543-MS
}

Intormal Report

UC.32

Issued: October 1976

\section{Computing in Support of Experiments at LAMPF}

by
R. F. Thomas
R. H. J.ppesen ${ }^{c}$
B. M. Preedom ${ }^{f}$
J. F. Amanna
S. S. Juhnson
R. J. Ridgeg
iH. S. Butler
W. K. McFarlane ${ }^{d}$
E. B. Shera
J. M. Gallup ${ }^{b}$
M. W. McNaughton ${ }^{e}$
H. A. Thiessen
R. E. Mischke
D. G. Perry

a Carnegie-Mellon University, Pittsburgh, PA 15213

b Lawrence Berkeley Laboratory. Berkeley, CA 94720

c University of Montana, Missoula, MT 59801

d Temple University, Philadelphia, PA 19122

e Case-Western Reserve biniversity, Cleveland, $\mathrm{OH} 44106$

$f$ University of South Carolina, Columbia, SC 29208

9 University of California at Los Angeles, Los Angeles, CA 90024

\section{scientific laboratory \\ of the University of California \\ LOS ALAMOS NEW MEXICO 87545

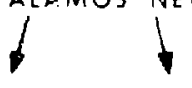 \\ An Alfirmative Action/Equal Opporsumity Emplnyer}


This woik was supported by the US Energy Research and Development Administration, Division of Physical Research.

Printed in the United States of America. Availabie from National Technical Information Service

U.S. Department of Commerce

5285 Port Royal Road

Springfield, VA 22161

Price: Printed Copy \$4.00 Microfiche \$2.25

Thin report wus perpared an an account of work upasuored

bo the liniled St stem Ciovernment. Neither the liniled Silale:

ministrulion. nor ans of the ir employere, nor sove of their con.

crustorn. eubrontrartork. of thris empiovees, makes an;

warraniy, exprews or implied, or ansumpans lecell liabil ty or

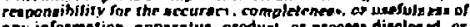

env information. apparatus. produrt, or procese diesloc d. or

rights. 


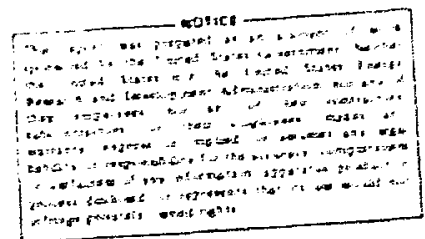

TABLE OF CONTENTS

ABSTRACT 1

1. PURPOSE OF THE STUDY AND PARTICIPANTS 1

II. SIMMARY OF RECOMMEMDATIONS 2

III. INTROOUCTION 3

IV. LAMPF RESPONSIBILITIES FOR DATA PROCESSING 4

A. Design of Hardware and Software 4

B. Development of Software 5

C. Checkout of Detectors and Electronics 5

D. Production of Data 5

E. Quick-Look Processing and Verification of Data 5

F. Bulk Analysis 6

G. Comparison with Theory 6

H. Archival Storage 6

1. Division of Processing Between LAMPF and
Other Institutions

3. Changing Needs 7

V. EQUIPMENT STANDAROS 7

A. Advantages of Continuing with the PDP.-11 8

1. Modularity and Flexibility 8

2. Maintenance and Spares 8

3. Software Support 8

4. User Training and Familiarity 9

B. Conclusion and Recommendation 9

VI. RECOMMENDED COMPUTER CONFIGURATION 10

VII. COMPUTERS FOR DATA ACQUISITION ANO PREPARATION OF EXPERIMENTS 11

VIII. DATA ANALYSIS 11

A. LAMPF's Alternatives for Data Analysis 12

1. The Large Computer 12

2. The Medium-Sized Computer 13

3. One or More Large PDP-11':; 13

B. Recommendation 14

IX. DATA STORAGE 14

A. The Present Situation 14

B. Recommendations 15 
X. LAMPF SOFTWARE SUPFORT 16

A. Software Support Personnel 16

B. Operating Systems and Data-Acquisition Programs 17

C. Dissemination of Software Information to Users 17

D. Summary of Recommended Level of Support and Distribution of Effort 18

X1. COMPUTER MAINTENANCE 18
A. Costs
8. Quality
18
C. 24-Hour Coverage
19
D. User Responsibilities 21
E. Recomrendations 21

XII. COMPUTER NETHORKS 22

A. Discussion 22

B. Recommendation 23

XIII. ADVANCTOD TECHNOLOGY 23

XIV. REMOTE JOB ENTRY TERMINAL 24 


\title{
COMPUTING IN SUPPOPT OF EXPERIMENTS AT LAMPF
}

by

R. F. Thomas, J. F. Amann, H. S. Butler, J. M. GalYup,

R. H. Jeppesen, S. S. Johnson, H. K. McFarlane,

M. W. McWaughton, R. E. Mischke, D. G. Perry, B. M. Preedom, R. J. Ridge, E. B. Shera, and H. A. Thiessen

\begin{abstract}
This report documents the discussions and conclusions of a study, conducted in August 1976, of the requirements for computer support of the experimental program in medium energy physics at the Clinton P. Anderson Meson Physics Facility.
\end{abstract}

\section{PURPOSE OF YHE STUDY AND PARTICIPANTS}

This report documents the recomendations of a study group appointed to assess the current status of computing factlities at the Clinton P. Anderson Meson Physics Facility (LAMPF) for use in direct support of the experimental program and to recomnend policies and courses of action for the future which will help in making the most effective and efficient use of the resources of LAMPF and its user community.

The participants in this study were: J. F. Amann (for R. A. Eisenstein), Carnegie-Melion University; H. S. Butier, LAMPF; J. M. Gallup, LBL; F. H. Jeppesen, Universit.y of Montana; S. S. Johnson, LAMPF; W. K. McFarlane, Temple University; M. W. McNaughton (for P. R. Bevington), Case-Hestern Reserve University; R. E. Mischke, LAMPF; D. G. Perry, LASL; B. M. Preedom, University of South Carolina; R. J. Ridge, UCLA; E. B. Shera, LASL; H. A. Thiessen, LAMPF; and R. F. Thomas, LAMPF.

Questions or comments regarding this report should generally be addressed to R. F. Thomas or H. S. Butler. 


\section{SUMMARY OF RECOMMENDATIONS}

The following is a summary of the recommendations made by the study group. Each topic is discussed at greater length in subsequent sections of this report.

1. For the present, LAMPF should continue to use the PDP-11 as the standard LAMPF computer. The LAMPF computer support staff should continuously monitor the characteristics of computers offered by the various manufactureis for developments which might significantly alter the relative advantages of the PDP-11 with respect to other types of computers. In the event thit such a development should occur, a change in the standard should be made only as the result of a formal study by a representative committee of LAMPF users.

2. A standard configuration for a LAMPF data-acquisition computer, suitable for running the LAMPF-supported data-acquisition coftware, is defined as iollows:
a) PDP-11 CPU with all options required by $\mathrm{RSX}-110$
b) $96 \mathrm{~K}$ words of memory
c) Floating point unit (software compatible with FPI1B)
d) Two RKO5 disks (or software- and media-compatible equivalent)
e) Tektronix 4010 (or software-compatibie equivalent)
f) Magnetic tape unit (compatible with DEC support software)
g) DECwriter (or equivalent)
h) MBD (CAMAC branch driver)

3. In order to accommodate anticipated growth in the data analiysis comput. ing needs, and täking into account limited computing resources expected to be available from the LASL Central Computing Facility, LAMPF should provide, as soon as possible, two PDP..1l's configured for maximum data-analys is capability to be dedicated to chis function. A permanent building to house the dataanalysis facility should be provided. In order to accommodate expected continuing limitations on C.CF usage, funds, in the amount of $\$ 300,000$, should be budgeted for a major enharicement to this facility in FY-1979.

4. The only practical medium for storage of data from experiments is industry-compatible magnetic tape. For future computer purchases, LAMPF should adopt a new tape standard. The new standard drive should be 9-track and switchable for either 800 or 1600 bits per inch. In order to provide a facility at LAMPF for converting between the old 7-track tapes and the new 9-track standard, a 9 -track, $800 / 1600$ bpi unit should be added to the LAMPF Terminal Computer. When 9-track operation has become a practical reality, most existing 7 -track TU10 tape units should be converted to 9-track operation.

5. Development and support should continue for data-acquisition program Q. A data-acquisition system consultant should be available for assignment to each experiment for consulting and specializad programing. The effort devoted to education and documentation should be increased significantly. In order to provide for these needs, the staff devoted to experimental software support should be increased to a level of at least five FTE's. 
6. The quality of maintenance on Jata-acquisition computer systems is unsatisfactory. LAMPF should have as a goal a level of service which includes single-party system responsibility, engineering and software backup for the maintenance offort, engineering review of maintenance problems, design and installation of engineering changes to make equipment more reliable, and review, design, and implementation of diagnostic software. As a first step in achieving this goal, LAMPF should hire an electrical engineer, experienced in computer system hardware and software, to oversee the maintenance services, and to help devise both engineering and administrative changes which wiil improve the maintenance and reliability of the equipment.

7. Due to the limited resources of $L A M i F$, twenty-four hour maintenance coverage for the data-acquisition computer systems should be dropped. A new maintenance contract which provides seven-day-per-week coverage with a guarantee that problems reported during the day will either be corrected or worked on for the first half of the night should be negotiated.

8. An engineering study should be undertaken, in collaboration with other laboratories, if possible, of a special PDP-11 peripheral device for histogram accuriulation. Such a device, most likely based on a combination of mirroprocessor and low-cost memory technology, offers the possibility of significant improvements in data-analysis capability, bo:h off-line and on-line.

9. In order to provide a basis for the sharing of expensive peripherais znd a common file system, the feasibility of comunications links among the POP-11's should be established. Commercially available hardware and sofiware should be procured and instailed for evaluation with respect to performance, reliability, and operating costs. Future implementation of a network should be guided by this evaluation.

10. The LOB CDC UT-200 terminal to the CCF NOS machine should be replaced by ä minicomputer emulator.

11. Each experimental team or facility should designate a computer system manager to be responsible for coordinating the support of the data-acquisition computer system assigned to the ream or facility.

12. LAMPF should continue to maintain a high level of expertise in digital systems technology and to pursue developments in this area which may lead to iilprovements in experimental apparatus. Collaboration ifi this fieid with other laboratories should continue to be encouraged.

\section{INTRODUCTION}

In the summer of 1970, there tock place a design study for data-acquicition systems at LAMPF (see LAMPF Data-Acquisition System, LA-4504-MS, August 1970). While the general directions outlined in that study have been followed, the plan has been modified through the years to adapt to changing conditions at LAMPF and in the cornputer industry. The detailed development of LAMPF experimental areas has been different from that envisioned in 1970, aria the economics of the use of small and medium sized computers has undergone significarit changes. In addition, experience has accumulated in the use of the existing data-acquisition sy'stems at LAMPF. 
In view of these circumstances, it was decided to conduct a study at LAMPF during August 1976 to review the currenc status of both data-acquisition and data-reduction facilities for experimental support. The membership of the study group consisted of LAMPF support personnel, LAMDF physicists, and outside users. The LAMPF users were chosen from those who have had recent experience with the data-acquisition systems.

The study group met for a total of five days on August 4,5 , and 6, and agizin on August 19 and 20,1976 . The interval between the two sessions was used to prepare position papers and obtain additiona? information felt to be relevant to the final recommendations.

While the finite resources of MP Division were considered in the recommendations, it was decided not to restrict the recommendations in such a way as to assure that they could be satisfied by amounts presently budgeted for this type of equipment. Instead, the needs of LAMPF have been described. The time scale on which they are satisfied must, of course, depend upon the allocation of resources hetween these and other needs of the facility.

The recommendations assume that the HRS and EPICS experimental facilities will be brought into operation according to present schedules, both to be operational in FY-1978, that beam intensity will be gradually increased as projected, that there will be no extended accelerator shutdowns, and that ctherwise there will be no major new experimental facilities added in the next three to five years. They also assume that there will be little or no growth permitted to LAMPF in the usage of the LASL Central Computirig Facility in this period. This last assumption, which is diametrically opposite to that made in the 1970 study, has particular impact on the problems of data reduction.

The sections which follow discuss the various issues addressed by the study group, the recomendations which resulted, and the reasoning which led to them.

\section{LAMPF RESPONSIBILITIES FOP. DATA PROCESSING}

One must consider all the steps in the processing of data from time of approval of an experiment until the results are published in a journal. In Fig. I, the steps in this process are illustrated for a typical experiment. In order that experiments be completed as quickly and correctly as possible, it is desirable for LAMPF to provide some assistance to experimenters at each stage in the process.

A. Design of Hardware and Software

At this stage, the major requirement is for consultation. Users need to know what standard designs and programs are available, what operating systems and programing languages are supported, what mediums for storage and interchange of data and programs can te utilized, and what computer system resources are likely to te needed and available for data taking and analysis. 


\begin{tabular}{|c|c|}
\hline $\begin{array}{c}\text { A Design of Hardware } \\
\text { and Sof tware }\end{array}$ & $\begin{array}{c}\text { E Quick Look, Verifi- } \\
\text { cation of Data }\end{array}$ \\
\hline $\begin{array}{c}\text { B Development of } \\
\text { Sof tware }\end{array}$ & F Bulk Analysis \\
\hline $\begin{array}{c}\text { C Checkout of Detectors } \\
\text { and Electronics }\end{array}$ & $\begin{array}{c}\text { G Comparison with } \\
\text { Theory }\end{array}$ \\
\hline $\begin{array}{c}\text { D Production of } \\
\text { Data }\end{array}$ & H Archival Storage \\
\hline
\end{tabular}

Fig. 1. Steps in processing data for an experiment.

\section{B. Development of Software}

Users who bring their own computers to LAMPF ordinarily prepare the required software at their home institutions. Those who use the standard computers at LAMPF need access to machines in advance of their runs in order to familiarize themselves with existing software and to develop the software which is special to their experiments. Standard software can minimize the total time spent in this state by both classes of user, but only if adequate documentation and consulting is provided.

\section{Chechout of Detertors and Electronics}

Typically, several months for each experiment are spent in this stage. An essential part of this work cannot be accomplished without a beam and computer. Those users who plan to use the LAMPF computers are particularly sensitive to this problen since these computers are tightly scheduled and are hcavily comitted to experiments that are already running. Adequate computer time, space, arid beam must be devoted to this work or poteritial production time will be diverted to checkout. Good interactive software systems can minimize the time spent in the checkout stage.

\section{Production of Data}

During production, the major resporsibility of LAMPF is to ensure smnoth operation with minimal interruption and reliable data recording. Good maintenance of the computer systems is particularly important. Reliable, crashresistant software and rapid response to problems also make major contributions to this phase of experiments.

\section{E. Quick-Look Processing and Verification of Data}

During production, it is frequently necessary to process a fraction of the data to demonstrate that the apparatus is working and to get information used to direct the course of future data taking. This need can be met by providing an off-line facility with rapid turn around. In some cases, it may be possible to process a fraction of the data using the data-acquisition machine on a time-available basis, if sufficient system resources are available. There 
is no substitute, however, for reading tapes on a different drive from the drive on which they were written to prove that the data recording has been accomplished successfully. Some experiments require access to a larae-scale computer with rapid turn around to do this phase of their data processing. Remote terminals such as the UT200 can be useful at this stage.

\section{F. Bulk Anelys is}

Approximately half of the experiments done at LAMPF record such a large amount of data that it cannot be processed in a real-time mode. These large experiments may require many times as much processing time as recording time. Such experiments benefit from high-quality data recording, including highdensity tapes. It is LAMPF's responsibility to see that adequate analysis facilities are available and that efficient use is made of existing resources during data analysis. It is important that there be a good match between datarecording and data-analysis machines in order that the required processing be accomplished at minimal cost. Large computing facilities are adequate for this work if good tape-reading equipment, high-capacity data busses, good bit- and byte-manipulation commands, and large-capacity data storage facilities are available. Highly efficient, off-line replay, using software which supports hoth data acquisition and reduction, can eliminate many adoitional programs which have beer required for this work in the past. Some experiments, however, such as the parity conservation experiments, may require very sophisticated processing of each element of data which is not feasible on a smaller machine. It should be noted that things are sometimes learned only after analysis of a large amount of data which requirn reprocessing of all the data from an experiment. One must include a factor in bulk processing time estimates to account for this phenomenon.

\section{G. Comparison with Theary}

At this stage, large-scale computers are particularly impurtant, since complicated high-precision, nonlinear calculations may be required. Since it is difficult to write general software for this stage of processing, computer operating systems which are designed for a high degree of sof tware portability are particularly important. If large computer resources are limited, the highest priority for their use should be reserved for work which can be accomplished in a reasoriable way only on large machines.

\section{H. Archival Storage}

Since experimental data cannot easily be reproduced, it is nurmal practice to save data tapes for two to five years to cover the possibility of questions arising which require reprocessing to resolve. The cost of archival storage is significant and cannot be overlooked. High-quality, high-density data recording can make an important impact on the cost and difficulty of the required storage.

1. Oivision of Processing Between LAMPF and Other Institutions

The processing of data after the production stage is divided between LASL and other institutions along lines of the location of major participants and sources of funding. The teaching responsibilities of university users require 
that they operate with half or more of their time spent at their home institutions. Software development and data processing at computers away from Los Alamos is a natural consequence. On the other hand, the needs of the LASL Staff require that an effective way of processing data be provided at Los Alamos as well. Funding and availability of processing time can significantly alter the fraction of computing done at. Los Alamos. Estimates of the fraction which would be done at Los Alamos vary between $15 \%$ and $70 \%$, depending on the conditions of availability and access.

J. Changing Needs

In projecting to the future, it must be recognized that developinent of hardware and software for many of the experiments now running was done on computers which were otherwise idle during the Great Shutciown. The next generation of experiments will require more facilities, if they are to be at the same state of readiness when their turn for production comes. The increase in beam current will also result in an increased amount of data. Finally, two major facilities (HRS and EPICS) ari just starting to come on line. The data produced by these spectrometers will have a major impact on the LAMPF dataprocessing workload.

\section{EQUIPMENT STANDARDS}

The 1970 summer study recommended that a single family of computers be selected to serve as the standard LAMPF data-acquisition computer. In a competitive solicitation conducted subsequent?y, the PDP-1l was selected. At that time $i t$ was the lowest cost small computer offering the required features. Since then, the market in sme?! computers has changed a great deal. The PDP-11, as anticipated, has been augmented in many ways, but other manufacturers also offer good quality compinters at very competitive prices. The benefits which were expected to result from standardization have been realized, probably to a greater degree than was foreseen. This success has come in part from the development of succeeding models of the PDP-11 CPU, which have offered improving price/performance ratios and remained comparable to small computers offered by competitors; it has come partly from the combination of modularity and comercial success of this particular machine, which has induced rival firms to offer plug-compatible components for PDP-11 systems; and it has come from the extensive body of software available from both commercial and noncommercial sources for this family of computers.

The standard has been applied with some fiexibility. In particular, system components such as memory and peripheral devices have been purchased from sources of plug-compatible equipment in order to take advantage of the lower costs of such equipment while still preserving the very substantial advantages of common software and interface standards. Even ihese purcliases of piugcompatible equipment bear a cost in terms of maintenance support, however, and the savings gained in equipment costs musi be weighed against the added costs of spares and training whenever a decision is made with regard to a particular item. 
1. Modularity and Flexibility. The PDP-11 is a highly modularized zystem. Since many of the LAMPF c'sputers are administered as a part of LEEP (LAMPF Electronic Equipment Pooij, advantage is taken of this modularity to reconfigure systems from time to time by adding and subtracting memory arid peripherals. Enhancements to existing computers are generally easy to accomplish and economical. Although the practice has some drawbacks, it is possible to troubleshoot and maintain systems by interchanging modules among different computers. The principal advantage, however, is the possibility of reconfiguration of systems by transierring components from one computer system to another to meet the changing requirements of the experimental program.

If one computer of a different make were to be introduced, there would be no possibility (in all likelihood) of exchanging memory or peripherals with the existing PDP-il's. The present situation with the existing SuperNOVA is an excellent examp? make were acquired, the situation would be somewhat better, but there would still be less flexibility in the assignment of resources than with complete compatibility. In general, the introduction of one or more different makes of computer into the inventory would incur costs associated with limiting the potential for reuse or multiple use of equipment. This property of the standard probabiy results in approximately $5 \%$ better utilization of equipment than would otherwise be possible.

2. Mainterance and Spares. Multiple units of identical equipment offer savings in maintenance by permitting training of maintenance personnel to be amortized over more pieces of equipment, permitting spare parts inventories to be spread over more units, permitting greater specialization and consequently greater expertise on the part of maintenance personnel. For the multiplicity of units and the size of the systems involved at LAMPF, it is even possible to have spares of major subsystems (such as disk drives) so that a computer system can be returned to service by replacing a complete subassembly. In addition, there are at present two sources of maintenance for PDP-11's at Los A1amos: Digital Equipment Corporation and the minicomputer maintenance group in the Electronics Division. Many cornpetitive systems do not have maintenance personrie: nearby.

3. Software Support. A11 of these computers require software support. Experimenters provide their own support to varying degrees. Some are completeiy independent; others require, or request, extensive help. Over the past few years, LAMPF support personnel have produced subroutines and utility programs for the PDF-11's. These programs represent approximately three man-years of effort. In the last two years, they have implemented a general data-acquisition package of programs under the operating system RSK-11D. This system is stil1 deveioping, aithough it is now in use on several machines. At present, it represents approximately four man-years of effort. Experimenters aiso produce programis which must be transferred from one computer to another. Al though standard languages help in going from one type of machine to another, this transfer is accomplished with the least cost in time and troub?e between computers which are identical. 
A second issue which falls under this general category is installation, maintenance, and corisultation with respect to the manufacturer-supplied software. These activities absorb the equivalent of approximateiy one staff member full time on the existing PDP-11's. To some extent, the effort depends on the number of computers and the number of users, but the expertise on which the activity is based is acquired at considerable cost in time and is reusable for each machine. The duplication of this activity for a different type of machine would represent significant continuing cost of its support. It is estimated that the cost to frovide the level of suftware suppori and service now available on the PDP-17's would be five man-years plus one FTE for cuntinuing support.

4. User Training and Familiarity. It is a burden on the users of these machines, whose primary interest is in their experiments, to acquire a sufficient level of expertise to operate them and program them. The cost to a single experimental team to provide the necessary software support for an experiment is estimated as follows:

- To become familiar with a new type of computer and produce all necessary software, using only manufacturer-supplied facilities: two man-years.

- To learn and utilize a more speciaiized data-acquisition software system such as program $Q$, now in use at LAMPF: six man-months.

- To use a wel1-supported data-acquisition software system with a maximum of enhancements and features: one man-month.

Thus, the potential cost to the LAMPF user community of a change to a different standard or simple abandonment of the PDP -11 standard is very high.

B. Conclusion and Recommendation

The current cost to LAMPF and its users of utilizing and supporting the existing computers exceeds the typical annual capital expenditures for computer equipment by a factor of four to five. The purchase of a single computer of a different type would result in additional support costs which would almost: certainiy exceed the cost of the computer. A change to a different standard which would then be adhered to for future purchases is estimated to increase the cost of overall computer support and utilization by $50 \%$.

It is recognized that developments may occur in computer technology which could make a change in the standard cost-effective, despite the factors cited above. The development of high-performance, low-priced machines with a longer word length, for example, might be sufficient to justify such a change.

For the present, however, LAMPF should continue to use the POP-11 as the standard LAMPF computer. The LAMPF computer support staff should continuously monitor the characteristics of computers offered by the various manufacturers for developments which might significantly aiter the relative advantages of the PDP-11 with respect to other types of computers. In the event that such a development should occur, a change in the standard should be made only as the result of a formal study by a representative committee of LAMPF users. 
Since the cost of software support and user software development makes up such a large part of the cost of data-acquisition computer usage at LAMPF, every reasonable step should be taken to simplify these activities and reduce the costs. The steps which can be taker include the adoption of a single manufacturer-supplied operating system and the establishment of a uniform mininium computer configuration capable of supporting the operating system and the standard LAMPF software and providing adequate additional rescurces so that a user is not required to spend his effort in tailoring his software to fit into limited storage or disk space.

Consequently, the following 7 ist is recommended as the minimum standard configuration for a LAMPF daia-acquisition computer.

1. POP-11 CPU with al1 options required by RSX-110. (RSX-11C is the current7y supported manufacturer-supplied operating system at LAMPF. The options required include a line clock and memory management. The requirements can be met with the PDP-11/34, PDP-11/45, PDP-11/55, or PDP-11/70 mode1s.)

2. $96 \mathrm{~K}$ words of memory. (For a single-user system, this quantity of memory should satisfy typical requirements. Larger quantities of memory may be requireú for multi-user systems or special applications such as large is istogram arrays.)

3. Floating point unit. (The currently supported FORTRAN compiler is F4P because of the great improvement in object code performance which it provides. The unit required by this compiler is the FPIIB, FPIIC, FPIIAU, or other software-compatible unit.)

4. Two RKO5 disks or equivalent. (For a single-user system, this quantity of disk storage should satisfy typical requirements. For a multi-user system, at least one additiona? RKO5 unit is needed. The requirement can be satisfied by the plug-compatible units of various suppliers, including those which have a single removable cartridge combined with a nonremovable disk. In order to facilitate the exchange of information among computers, however, the removable cartridge(s) should be capable of being read and written on the DEC RK05.)

5. Tektronix 4010 or equivalent. (The graphics software depends on a device of this type. The requirement is met also by the Taktronix models $4012,4013,4014$, and 4015 , but not by the model 4006. )

6. Magnetic tape unit. (Plug-compatible units which are compatible with manufacturer-supplied tape software meet this requirement. For new equipment 9-track, 800/1600 bpi units are recommended.)

7. Hardcopy console terminal. (On the PDP-11, this unit is normaliy the DECwriter. Other equipment which presents an identical software interface can be substituted.) 
8. BiRa MBD. ( $1 \mathrm{~K}$ words is the minimum recommended memory size. Special applications may require additional memory. The data-acquisition software requires this interface to CAMAC.)

\section{COMPUTERS FOR DATA ACQUISITION AND PREPARATION OF EXPERIMENTS}

HRS and the main beam channels in Area $A$ are all supplied with LAMPF data-acquisition computers; yet the constraints imposed by computer availability continue to impact the scheduling of experiments. Two circumstances account for most of this impact:

- Not all the computers meet the minimum recommended configuration. in particular, the PDP-11/20's cannot support the standard software now in use.

- There is essentially no rescurce available for checkout of software and electronics for upcoming experiments. The first round of experiments after the Great Shutdown enjoyed the luxury of using the data-acquisition computers, which otherwise would have been idle, for software development and hardware checkout. For experiments scheduled subsequently, the situation is much more difficult.

If computers are not available to support the needs of experimenters, the productivity of LAMPF is limited as a result. The situation is especially serious for the LEP channel. It is the most heavily subscribed channel, which means that inefficiency in its use must be minimized. Beam time is consumed by equipment and software checkout which could be done on an auxiliary facility. Certain experiments must be scheduled in sequence when the availability of needed equipment would have permitted them to be scheduled in parallel.

In order to minimize shese problems and to make the most efficient use of the accelerator, it should be a goal of LAMPF to provide a stanciard computer for each experimental beam port and to provide computers for testing of hardware and software for experiments in preparation. In accordance with this goal, the most urgent requirements are for two standard computers to support experimental checkout. If the first of these is located at the test channel, it will serve the needs of the upcoming experiments at LEP and other users as we11. Additional needs are for one standard computer to replace the existing PDP-11/20 at $\mathrm{P}^{3}$, memory to bring al1 PDP-11/45's to a minimum of $96 \mathrm{~K}$ words, and KS90 tape controllers to replace existing LBL tape controllers.

\section{DATA ANALYSIS}

The event-by-event type of experiment is the most consuming of computing resources. In order to estimate the potential magnitude of future requirements in this area, a survey was taken of some 30 event-by -event experiments. These experiments involve the analysis of approximately 1800 magnetic tapes at the LASL CCF. An additional 800 (30\%) have been analyzed at LAMPF on POP-11's or taken to the users' home institutions for analysis. 
The experiments which were analyzed at CCF tended to have heavy LASL participation, while those analyzed on PDP-1l's or at other facilities tended to be those by groups witi! little or no LASL participation.

The cost of the analysis at the CCF has varied from $\$ 20$ to $\$ 100$ per tape $(0.2$ to 1.0 COC 6600 CPU hour per tape). The variation in cost depends both on the complexity of the analysis and the number of passes made through the data. By analyzing data on a PDP-11 and scaling the results by the ratio of PDP-il and CDC 6600 FORTRAN speeds an estimate of the cost per tape has been made for the types of experiments to be done at EPICS and HRS. This estimate yields a value of $\$ 18$ to $\$ 28 /$ tape/pass (or 0.18 to $0.28 \mathrm{CPU}$ hours/tape/pass). A total cost estimate based on two passes through the data indicates that average costs of $\$ 30$ t. $\$ 50$ per tape can be expected. The entire MP-Division budget at CCF for the last year $(\$ 117,000)$ would analyze 2500 to 4000 such tapes.

Predictions as to the amount of data to be collected in the future vary by a factor of two or more. For the channels already in operation, the increased beam intensity in the future indicates that up to five times as many tapes may be generated per year in the future as have been in the past. Thus, a number of 5000 tapes per year is not unreasonable as the estimate for all channels now in operation.

For HRS and EPICS, a guess of one tape per hour for each line is a reasonable upper limit for the average rate of tape generation during production. If $60 \%$ of the calendar year is devoted to "production" beam, 5000 tapes per facility per year will be produced. This estimate seems high, but since 500 tapes per experiment is not an inordinately large number, it would only require 10 such experiments per year on a channel to produce 5000 tapes. A. low estimate is 250 tapes per experiment $\times 10$ experiments per year $=2500$ tapes per year. Thus, HRS and EPICS together may produce 5000 to 10,000 tapes per year, with a total for LAMPF as a whole of from 10,000 to 15,000 tapes per year. At $\$ 30$ to $\$ 50$ per tape for full analysis, this level of activity implies a cust of $\$ 350,000$ to $\$ 750,000$ per year, if all were analyzed at CCF. This result requires at least tripling MP-Division's current CCF usage, but the Director's Office has indicated no substantial increase in allotment will be available in the near future. Also, in the most readily accessible form of storage at CCF, Hydra Photostore, 10,000 tapes would nearly utilize the entire on-line storage capability in a single year. Indeed, simply the cost of purchasing 10,000 to 15,000 tapes per year is $\$ 100,000$ to $\$ 150,000$ per year.

A. LAMPF's Aiternatives for Data Analysis

Since CCF will be unable to accommodate any increase in computing load from LAMPF, one must look at the possible alternatives for analyzing the data produced. In this section, three al ternatives for LAMPF data analys is computing are examined: 1) buy a very large machine such as a CDC 6600 or IBM $360 / 195,2$ ) buy a medium-size machine such as a DECsystem 10 or 20 , or 3) buy one or more smaller machines such as the PDP-11/45 or $11 / 70$.

1. The Large Computer. Since some fraction of data analys is (such as theoretical curve fitting) involves the running of very large codes, it would be useful to have a computer which would run these codes. Also, the speed of these machines is an advantage for compute-bound jobs. 
The acquisition of such a large computer, however, has several disadvantages. First of all, since CCF wil? continue to provide some ability to run large codes, it is not obvious that such a large machine is necessary. It is also not certain how much of off-line analysis is compute-bound as compared to how much is $1 / 0$-bound.

A second consideration must be the very high purchase and maintenance costs. (CCF spends approximately $\$ 100,000$ per year on maintenance for one 6600 CPU.) In addition, large machines require carefully controlled environments, which mean substantial costs for a building and its maintenance. It is also estimated that operating costs would be very high; at least six to eight full-time personnel would be required to operate and manage such a facility.

Finally, much of the software used for data acquisition would have to be rewritten for data analysis.

2. The Medium-Sized Computer. As in the case of the large computer, the ability to run large codes makes such a machine attractive, especially at its lower capital cost (about $\$ 500,000$ ). Unfortunately, this alternative suffers from many of the same disadvantages as the large computer, although on a smaller scale. Operating costs (CTR has two staff members to operate their DECsystem 10), high maintenance (hardware not compatible with LAMPF's present computers), and lack of software compatibility are all drawbacks of such a machine. In addition, presently available machines in this class operate at a speed comparable to that of the highest-performance PDP-11's.

3. One or More Large PDP-11's. Small computers of the PDP-11 class appear to be particulariy attractive as a means for general data reduction. Although the 16-bit word length limits the size of a program which can be managed, by far the largest portion of the data produced at LAMPF, particularly the data to be produced at EPICS and HRS, could be reduced on such a small machine.

This alternative is attractive for several reasons: 1) the low cost would permit purchase of several machines for the same investment as a mediumor iarge-sized computer, and ease of networking with the on-line machines would allow some pooling of peripherals, 2) since LAMPF already has several PDP-11's, maintenance spares are already on hand, and some maintenance expertise is presently zvailable at LASL, and 3) past experience with PDP-1l's indicates operating costs are about $\$ 20,000$ per year per system for systems management and consulting and about $\$ 15,000$ per year per system for maintenance.

The use of a PDP-11 has additional advantages in that the software used for data acquisition could be used directly on the analysis machine with little or no modification, thus saving a considerable amount of software development time which would be needed if that data were analyzed on another type of machine. This portability of software is a very strong reason for doing anaiysis on the same type of computer on which the data was taken. As has been mentioned previously, the effort required to produce data-acquisition or analysis software may range from several man-years to a few man-months, depending on the support and facilities available. Thus, it is clear that much labor can be 
saved by using such software as already exists on these small machines. Furthermore, all the special feacures that one adds to any system to tailor it to the specific needs of the experiment would not need to be redone if analysis were done on the same type of machine.

The speed of the existing PDP-11/45's at LAMPF, for a program small enough to fit, has been measured to be one-fifth to one-sixth that of a CDC 6600. Other benchmark studies indicate that commercially available hardware enhancements, such as cache memory and a faster floating point unit, can approximately double the speed. A disadvantage of the PDP-11/45 is the $1 \mathrm{imi-}$ tation on total memory to $124 \mathrm{~K}$ 16-bit words. The PDP-11/70, for which cache memory and faster floating point are standard, can have up to one million words.

Another disadvantage of the PDP-11's is the small address space (32K words) for any one program. Several factors make this limitation less critical. Good intertask communication allows many tasks to use the same data. Facilities in new operating systems offer improved ways to cope with this limitation. Since much of the space required in many applications is for histograms, specialized software or haroware for managing histograms can increase the number of jobs which will fit in this space.

B. Recommendation

In order to accommodate anticipated growth in the data-analysis computing needs, additional facilities must be made available in the near future. Since little or no growth of LAMPF's use of the LASL Central Computing Facility is expected to be possible and since computers of the PDP-11/70 class offer a cost-effective solution for many of these needs, LAMPF should provide as soon as possible two such computers configured fa: maximum data-analysis capability to be dedicated to this function. A permanent building to house the dataanalysis facility should be provided.

If restrictions on LAMPF usage of the Central Computing Facility continue, the data-analysis facility will need to grow as the accelerator and experimental facilities become more productive. It is recommended that $\$ 300,000$ be budgeted for a major enhancement in FY-1979.

\section{DATA STORAGE}

A. The Present Situation

Taking account of cost and commercial availability of equipment, the required speeds, and the cost of the storage medium, there appears to be no viable, economic alternative to the use of industry-compatible magnetic tape for the recording and storage of data from LAMPF. Presently, there are 24 tape drives at LAMPF, including those belonging to MP-Division, CNC-11, and P-Division. Six of these are DEC TUlO drives; the remainder are IBM 729 drives. Of the $729^{\prime} \mathrm{s}$, nine are interfaced via the LBL-designed controller which operates through CAMAC, and ten are interfaced via the newer Kinetic Systems Model 90 controller. The tapes written by the TU10, LBL, and KS90 
controllers are all formatted differentily, so that tapes written by one controller can be read with another only with software to do the necessary reformatting. Presently, all tape drives write 7-channel tapes at 556 or 800 bpi.

The tape systems now in use present several problems. The variety of formats written by the various controllers (in their most efficient modes of operation) reduce the usefulness of magnetic tapes as a medium for information interchange among the computers. The information density on the tapes is a relatively low 32,000 to 33,000 bits per inch (taking into account all seven tracks in the recording modes used). The LBL controllers do not present a standard software interface to the PDP-11. Hence, special handlers, special bootstrap code, and special diagnostic programs are necessary to use and maintain them, and operation of the tapes on the CAMAC branch highway steals time from experimental data acquisition and forces other MBD code to be less efficient.

The Central Computing Facility at LASL currently encourages 7-track tape usage. Only one NOS machine, which can be accessed only by cleared users, has access to 9-track drives. Hence, any 9-track data tapes to be analyzed at C.CF are usually copied to 7-track first. CCF drives currently read 556 and 800 bpi tapes. The CDC tape drives have had a greas. :umber of reliability problems, and C-Division is currently studying how to move to faster, better tape drives in the future. Experience with the 9-track $800 / 1600$ bpi drives at CCF has been very good compared to the older 7-track drives. Many users" home computing facilities support 9-track drives, and the LASL 7-track standard creates serious compatibility problems for some users.

The C-Division tape library has reached full capacity. Consequently, the storage of the data tapes generated at LAMPF must be primarily a LAMPF responsibility.

\section{B. Recommendations}

For future computer purchases, LAMPF should adopt a new tape standard. The new standard drive should be 9-track and switchable for 800 or 1600 bits per inch. At the recommended 1600 bpi density, this new standard will provide a faccor of 4 increase in the information capacity of a tape. The 800 bji option will provide compatibility with older 9-track units. While even higher density units (6250 bpi) are available commercially for some computers, and such densities would offer some obvious advantages, the equipment is quite expensive and less commonly found in the average computer center, and it is not commonly used on machines of the PDP-11 class. It is felt that the effort and expense necessary to implement a standard based on this technology is not justified for LAMPF at present.

The existing IBM 729 tape systems should be retained as long as they can be maintained in satisfactory condition. The cost of replacing all of these systems immediately with 9-track units is not justifiable. The CAMAC-based LBL tape contrallers should be replaced by $\mathrm{KS90}$ controllers, however, to provide a greater degree of uniformity in tape formats and to simplify the software, maintenance, and operational problems which these units now create. 
In order to provide a facility at LAMPF for converting between 7-track and 9-track formats, a 9-track $800 / 1600$ bpi tape unit should be added to the LAMPF Terminal Computer.

When 9-track operation has become a practical reality, most existing 7-track TU10 tape units should be converted to 9-track, 800 bpi operation. These units cannot be converted to $1600 \mathrm{bpi}$ operation, but this change would have the effect of doubling the data rates and information density on tapes written on these drives.

C-Division should be encouraged to increase support for 9-track tapes, especially on machines which can be accessed by uncleared users.

\section{$X$. LAMPF SOFTWARE SUPPORT}

At present, and in the past, the number of available personnel for software support of LAMPF users has been inadequate to meet the needs of users. In the absence of this support, each user group has written its own software, often with little communication with other users or with LAMPF software support personnel. The results of this situation have been much duplication of effort, heavy dependence of user groups on a single indispensable programmer, and waste of effort when this indispensable programer leaves and his program is abandoned since no remaining person understands it.

Also, the change of operating systems from DOS to RSX-110 with the subsequent lack of support for DOS forced many users'codes toward obsolescence, since the operating system was no longer modified to take advantage of new hardware or software needs. It is recognized that this situation is due to a lack of manpower and not to a lack of cooperative spirit on the part of LAMPF personnel.

\section{A. Software Support Personnel}

At the present time, there are only two progirammers whose primary job is the general support and consulting for the data-acquisition computers. The software support for these computers should be increased until the level of support is sufficient to allow MP-Division to assign a software consultant to each experiment in much the same manner as an engineer is presentiy assigned. One programmer might support as many as four experiments at one time. It is not anticipated that this programmer would write code for each experiment to which he is assigned. Instead, he would consult with those members of the experiment who are responsible for the data analysis and acquisition code. He would become familiar with the experiment and, in doing so, would be able to make recommendations which might improve the efficiency of the code being written. He would also be familiar with all recent developments in the system software and would aid in incorporating any changes into the data-acquisition code for the experiment. It is anticipated that he would write code for the experiment only in those cases where a special handler. special MBD code, or other nonstandard code is required. At least two additlonal programers would be required to begin this program. 


\section{B. Operating Systems and Data-Acquisition Programs}

The present standard for nperating systeris at LAMPF is RSX-11D. This will remain the standard operating system until such time as other developments in computer software make a change attractive from both the economic viewpoint and witn respect to new capabilities offered the user. Future developments in RSX-IIM which may allow tasks to occupy more than $32 \mathrm{~K}$ words of memory make such a change a pussibility. If changes in operating systems take place, every effort should be made to make these changes transparent to the general user, at least with respect to his data-acquisition program.

An attempt should be made to obtain coverage for LAMPF users under the Laboratory's multicopy software agreements with the Digital Equipment Corporation.

The data-acquisition software system developed and being maintained at LAMPF is a collection of tasks and subroutines known as "Q." It is anticipated that this system will remain the standard for LAMPF and will continue to be supported by MP-Division personnel. It is recommended that future versions of the data-acquisition program be upward compatible with the existing version.

The use by many groups of a single software package should increase communication and continuity and reduce the dependence of groups on a single programer. It is unlikely, however, that $Q$ will ever be able to satisfy all of the needs of all of the groups without continual modification. Minor "ad hoc" modifications to $Q$ routines could be made by the experimenters responsible for writing the 0-based data-acquisition system for their experiment while major "ad hoc" modifications are expected to require the help of the dataacquisition system consultant assigned to the experimen+

C. Dissemination of Software Information to Users

Since many of the LAMPF users reside at universities and places other than LAMPF, it is imperative that thoy remain auvised of changes in the standard software being used at LAMPF. The present Data-Acquisition Newsletter is of great help in circulating this information. The publication of the newsletter should be put on a regular schedule and the circulation of the newsletter increased to include all those who are concerned with data acquisition at LAMPF. (This could be done by having each principal investigator name those people in his experiment who should receive the newsletter.)

The problem of user education with regard to computer software and $Q$ could be dealt with by the preparation of training manuals for the LAMPF computers. It is suggested that these training manuals be at three levels of difficulty: 1) Primer, 2) Detailed, and 3) Technical.

The primer level would be addressed to the computer operator and to the "physicist-programmer" who is writing a data-acquisition program under $Q$ for the first time. The primer would list the command structure for the computer and would provide a "cookbook" means of getting on the computer and writing or running a task under RSX-11D and Q. 
The drtailed level of the training manual would be addressed to the "physicist-programmer" who is actively engaged in writing data-acquisition code. It would provide enough information concerning the task structure of $Q$ and of the operating system to allow the user to make efficient use of all the standard features of the data-acquisition program. The documentation at this level, however, would not provide sufficient information to make modifications to $Q$ or the operating system.

The technical level wol?d be at the level of the manuals written by DEC and need only be written for Q. These manuals would give a detailed description of $Q$ and would allow the experienced programmer to make modifications to $Q$ which might be necessary for his particular experiment.

It is anticipated that the equivalent of one person full-time is required to prepare manuals, courses, and other educational aids, in addition to compiling the LAMPF Data-Acquisition Newsletter.

D. Summary of Recommended Level of Support and Distribution of Effort

It is recommended that the LAMPF support for data-acquisition software, consulting, documentation, and education be increased from the present level of two FTE to five FTE. The effort should be distributed as follows:

-Enhancements for $Q$ and deveiopment of other general software. 2 FTE

- Consulting with users and development of special software 2 FTE for particular experiments.

-Preparation and maintenance of manuais, teaching courses, 1 FTE and publication of newsletter.

\section{COMPUTER MAINTENANCE}

A. Costs

The cost of maintaining the computer equipment associated with the LAMPF experimental data-acquisition system has risen steadily since the first two computers were received in February 1972. The records indicate that in FY-75, a total of $\$ 132,000$ was spent on computer maintenance. This period included six months of experiments--June to December 1974. During FY-76, the expenditures for maintenance were slightly over $\$ 200,000$. About $\$ 85,000$ went to DEC, who had a maintenance contract for the entire year. The remainder went to E-5, the LASL computer maintenance organization, must of it during the last six months of FY-76, when their operation was formalized by a contract. During this period, beam was delivered to Area $B$ about eight months and to Area $A$ about three months. Maintenance costs are incurred whether or not the beam is on, because experimenters use their computers for data reduction and set up of new experiments when beam is not scheduled.

The current picture of maintenance costs is best seen $b_{j}$ examining the costs for July 1976. Contract costs for this month totaled $\$ 27,500$, about 
$25 \%$ to DEC and $75 \%$ to $E-5$. This rate equates to an annual total of $\$ 330,000$. Indeed, a total of $\$ 360,000$ is budgeted in FY-77 for computer maintenance.

Since 12 of the 25 computers under contrart are under 24-hour/7-day coverage, the costs are especially high at preserit.

If the FY-77 total is not alarming enough, one has only to consider the cost of maintaining several more computers which may be purchased in the coming year. To appreciate the potential magnitude of these costs, consider the monthly maintenance fee for computers of various sizes. This information is given in Table I.

TABLE I

\begin{tabular}{|c|c|c|c|c|}
\hline ystem & DEC Costs & $E-5$ Costs & Total for 8-5 Coverage & Annual Costs \\
\hline $\begin{array}{l}\text { Temple } \\
\text { LEP } \\
\text { Terminal } \\
\text { EPICS } \\
\text { Biomed }\end{array}$ & $\begin{array}{l}533 \\
845 \\
712 \\
884\end{array}$ & $\begin{array}{c}\$ 456 / \mathrm{mo} . \\
572 \\
325 \\
775 \\
1055\end{array}$ & $\begin{array}{l}\$ 456 / \mathrm{mo} . \\
1105 \\
1170 \\
1487 \\
1939\end{array}$ & $\begin{array}{c}\$ 5,500 / y r \\
13,300 \\
14,000 \\
17,800 \\
23,300\end{array}$ \\
\hline
\end{tabular}

If the middle three computers are taken as representative, then the purchase of each new computer adds an average of $\$ 15,000$ arinually to the cost of maintenarice. Thus, the costs go up linearily with the number of computers or, more precisely, with the amount of equipment under contract.

B. Quality

At present, the maintenance responsiblity for most data-acquisition compucers is divided between Digital Equipment Corporation and the LASL minicomputer maintenance organization (E-5). Because the computers contain much equipment. not supplied by DEC and company policy forbids the maintenance of such equipment, there is no possibility of total reliance on DEC for systen maintenance. Since neither organization has complete responsibility for the typical system and since computers are susceptible to failures which are difficult to localize and diagnose correctiy, there is a natural, human tendency for each team to hope, and believe, that these most difficuit problems are the responsibility of the other team. Many prnblems go unresolved for longer beriods than they should because of this situation.

The maintenance contract with DEC includes one feature, the effect of which is not immediately apparent--the hierarchy of support. If a problem cannot be solved by local field service personnel, DEC will import a specialist from the district office. If the problem still does not yield, an expert from the regional office will be called in. In principle, someone from the factory rould be obtained. In any event, after each problem is solved, information about the failure goes back to the factory where it is studied with tre idea of devising an engineering change that will correct the problem or improve the reliability in all systems. In due course, the user, as part of his contrart, can have the appropriate engineering change installed in his system. 
This same hierarchy of support is not available in E-5. When the field technicians are unable to solve a problem their only recourse is to call in other field technicians. While there exists a staff of competent engineers and programmers in E-5, none of them are familiar with the LAMPF problems, and they are not used in support of the field service technicians.

E-5 prices are only $5 \%$ below DEC charges, and this differential could be viewed as the profit margin for a commercial company. Hence, it is argued that a capable engineer in E-5 should be available to support the LAMPF maintenance. He should serve as a high-level backup for maintenance technicians, study the field service reports, identify chronic problems, and work out appropriate solutions in cooperation with the vendors' engineering staffs. This kind of support would reduce the load on the maintenance technicians, thereby resulting in a net saving that would cover the engineer's time.

As a practical matter, this level of engineering support is required only for the non-DEC equipment, since engineering changes can be obtained from DEC for their equipment.

One further shortcoming of the present mainterance effort is the lack of good diagnostic soitware for certain devices. The review, maintenance, and implementation of such snftware is a necessary part of maintenance support.

\section{24-Hour Coverage}

During June of this year, 10 of the 24 computers were under 24-hour coverage. The cost of this extended coverage was $\$ 7,660$ out of a total maintenance cost of $\$ 26,450$, or $29 \%$. During July two more computers went under 24 -hour coverage, pushing the costs to $\$ 8,930$ out of $\$ 27,560$, or $32 \%$. One is led to question the value of 24-hour coverage, especially in view of the fact that dropping it would save over $\$ 100 \mathrm{~K}$ per year. The cost of $24-$-hour/7-day coverage is approximately twice the cost of standard 8-hour/5-day coverage.

During the months of May, June, and July, a total of 238 field service reports were filed by both maintenance teams. Of this total 24 , or $10 \%$, represented calls made outside the primary coverage of 8 to 5 , five days per week. Viewed in one way, $10 \%$ of the calls are costing $32 \%$ of the maintenance fee. The actual distribution of calls is given in Table II.

TABLE II

$\begin{array}{lc}\text { Time of Day } & \text { No. of Cal7s } \\ 0800-1700 \text { weekdays } & 214 \\ 1700-0800 \text { weekdays } & 20 \\ \text { Heekends } & 4\end{array}$

This distribution is probably a reflection of the fact the best hours for taking data are nights and weekends. Thus, the calls which were made were probably for serious failures, the kind which take the system off the air. 
Another way of looking at 24-hour coverage is from the "insurance" point of view. The operating costs for the accelerator are about $\$ 30,000$ per shift. Assuming 10 beam ports in simultaneous operation, the cost of operating one port is $\$ 3,000$ per shift or $\$ 375$ per hour. The cost of extending the maintenance coverage from 8,5 to $24 / 7$ is about $\$ 600$ per month for a typical configuration, or $\$ 1.08$ per hour. An insurance premium of $\$ 1.08$ to protect an investment of $\$ 375$ doesn't seem unreasonable. The argument has to be questioned, however, when the premiums reach $\$ 9000$ per month, as was the case in July.

The value of the service received after hours appears to be lower than that received during normal working hours, when the majority of the resources and personnel of the maintenance teams are available.

D. User Responsibilities

The users of the data-acquisition computers have certain obligations which they have not, for the most part, accepted. The major fault is failing to keep the counting houses clean. Dust, smoke, and miscellianeous detritus are allowed to accumulate in the rocns despite repeated warnings of the potential costs in computer reliability. Failure to heed these warnings is costing isers beam time and driving up maintenance costs. For example, a recent study compared the number of service calls on a computer system with system complexity. Computers which exist in cramped and dirty quarters logged a larger number of service calls relative to their complexity. On the other hand, computers in a clean, well-kept area had substantially fewer maintenance calls than would be expected by the size of that system.

A second fault of many users is their failure to identify a system manager for each experiment. This person should be responsible for assuring that the computer equipment is functioning (1iaison with maintenance teams), that the latest version of the software is installed (liaison with programing staff), that the premises are kept clean, and that no one makes unauthorized changes in the system. He should see to it that appropriate backup copies of files and system disks exist and that up-to-date listings and other documentation for the specialized sortware in use are available. The existence of such a system manager would give the support groups a point of sontact in extending services. Also, the system manager could be debriefed at the conclusion of the experiment for suggestions on how to improve the level of support from the resident staff.

\section{E. Recommendations}

LAMPF should have as a goal a level of service which includes singleparty sustem responsibility, engineering and software backup for the maintenance effort, engineering review of maintenance problems, design and installation of engineering changes to make equipment more reliable, and review, design, and implementation of diagnostic software. This level of support implies the existence of personnel with a high level of competence in both digital engineering and software to whom field technicians can turn for support and help with difficult or chronic problems. It also requires placing 
the responsibility for the proper functioning of the systems in the hands of a single organization dedicated to their support and having the technical competence to diagnose and resolve the problems which arise.

As a first step in achieving this goal, LAMPF should obtain the services of an electrical engineer, experienced in computer system hardware and software, to oversee the maintenance services and to help devise both engineering and administrative changes which will improve the maintenance and reliability of the equipment.

Twenty-four hour maintenance coverage for the data-acquisition computer systems should be dropped. In its place there should be an arrangement which provides 7-day/week coverage for critical systems during normal working hours, with an additional provision that work on a maintenance problem reported during the day will continue into the night, until $11 \mathrm{p.m}$. or midnight, for example. Such an arrangement should permit the maintenance teams to apply a maximum of resources to problems and at the same time assure users of attention within a reasonable time.

Each experimental team or facility should designate a computer system manager to be responsible for coordinating the support of the data-acquisition computer system assigned to the team or facility. This person will be responsible for assuring that the computer and software are functioning properly, that appropriate help is obtained in case of failures of either hardware or software, that the area is kept clean, and that documentation and backups are in order.

\section{COMPUTER NETWORKS}

A. Discussion

While much activity at LAMPF (hardware checkout and debugging, system software checkout, operation under special operating systems) requires dedicated use of a computer, many other activities can, in principle, be carried out in a multiuser mode. In particular, many of the tasks associated with the development and management of software can be carried out in a multiuser environment and at the same time consume a very small fraction of a computer's resources. This situation has been recognized in the installation of multiple terminals on several of the LAMPF PDP-li's, especially the LAMPF Terminal Computer, which is accessible not only from three local terminals but from two telephone lines.

An extension to the multiple-use, communications-based mode of operation is to establish links between computers. Such a network offers the possibility of concentrating certain resources such as the more expensive or rarely used peripheral devices at a single point, or a few points, but giving other computers relatively convenient access to them. A second potential benefit of such connections is the capability of supporting a common file system. Remote access to a large file system can substantially reduce, and in some applications el iminate, the need for local disk storage. Furthermore, in an environment such as at LAMPF, where a substantial amount of comnon software is in use on many different systems, the maintenance and management of the software can become a complex and time-consuming affair. A central file system can make this activity simpler and more efficient. A common file system with 
remote acsess from terminals or other computers can also be used effectively by experimenters in the analysis of reduced data, especially in the irteractive mode.

Software suppori and high-performance communications interfaces are now available commercially for the computers and operating systems in use at LAMPF. Hence, it appears that a network could be implemented with reasonable cost and effort.

Two factors have a decisive effect on the ultimate practical use of a network. The network hardware and software and any computers involved in providing services to the netwoik must have a very high degree of reliability. Also, the operation of the network must not consume an excessive amount of CPU time or memory in the computers connected to it.

B. Recommendation

While several potential benefits can be expected from the implementation of a computer network at LAMPF, they can be realized only if the quality and dependability are high. At present, there is no experience at LAMPF in this area except in the very specialized network associated with the central control computer. Commercially available hardware and software should be procured and instalied on two or more computers. If the performance of this system is satisfactory, the network can be expanded to include more of the data-acquisition and data-analysis computers as the needs and advantages become apparent.

\section{ADVANCED TECHNOLOGY}

The field of electronics is continuing to advance at a rapid pace. Certain developments in this field can be applied to the problems of data acquisition and data reduction to improve the speed or lower the cost of these activities. The two most dramatic of these developments at present are the continuing improvements in microprocessors and microcomputers and the diminishing costs of electronic devices generally. Lower costs permit the use of larger quantities of electronics and thus a greater degree of parallelism in equipment. The use of microprocessors permits the implementation of relatively complex processing more easily and economically.

T'e most obvious application of these advances in the field of physics is in the construction of faster, more powerful front-end electronics. A major part of the current work of the NIM Committee, which is composed of representatives from ERDA laboratories, universities, and other scientific laboratories, is directed towaró defining standard schemes of introducing greater processing power and parallelism into data-acquisition electronics. LAMPF should continue to maintain a high level of expertise in digital systems technology and keep abreast of applications of the technology to similar problems at other laboratories. Collaboration in this field with other similar institutions should continue.

Studies have indicated that as much as half the time involved in reducing certain types of data is spent in making entries into histograms. It is 
conceivable that a histogramming device, based on microprocessor and low-cost memory technology, could significantly speed up the rate at which such data is reduced. Since histogramming is such a common feature of data-acquisition and data-analysis systems, it is recommended that a design study be undertaken in $c^{7}$ laboration with sther laboratories, if possible, to determine the feasibili $y$ nd cost of constructing such a device to be interfaced to the PDP-11 cipl sers.

\section{REMOTE JOB ENTRY TERMINAL}

The only remote job entry terminal for batch jobs available for use by uncleared personnel is tne CDC UT200 located in the Laboratory-Office Buflding. This unit is old and worn out and should be replaced. it is recommended that it be replaced by a minicomputer system which can emulate the UT200 functions. 